\title{
Drift-diffusion models for the simulation of a graphene field effect transistor
}

\author{
Giovanni Nastasi ${ }^{1 *}$ [D and Vittorio Romano ${ }^{1}$
}

\section{"Correspondence:}

giovanni.nastasi@unict.it

1 Department of Mathematics and

Computer Science, University of

Catania, Viale A. Doria, 6, 95125

Catania, Italy

\begin{abstract}
A field effect transistor having the active area made of monolayer graphene is simulated by a drift-diffusion model coupled with the Poisson equation. The adopted geometry, already proposed in (Nastasi and Romano in IEEE Trans. Electron. Devices 68:4729-4734, 2021, https://doi.org/10.1109/TED.2021.3096492), gives a good current-ON/current-OFF ratio as it is evident in the simulations. In this paper, we compare the numerical simulations of the standard (non-degenerate) drift-diffusion model, that includes the Einstein diffusion coefficient, with the degenerate case.
\end{abstract}

Keywords: Graphene FET; Drift-diffusion model; Graphene

\section{Introduction}

Since its discovery, graphene has attracted scientists for its possible use in electronics due principally to the high electron mobility; moreover, the thickness of just one atom [2] could represent the ultimate miniaturization. In the last years, several graphene field effect transistors (GFETs) have been designed [3] with the aim to substitute the standard silicon metal oxide field effect transistors (Si MOSFETs). The main issue is due to the absence of gap in monolayer graphene, causing the ambipolar conduction in the device and, consequently, a very narrow current-off region. In addition, the physical features of the metal-graphene interface at the contacts are not completely understood because they are rather different with respect to the case of traditional semiconductors in which the Ohmic or Schottky conditions are usually appropriate. The considerations above make to conceive GFETs a very challenging target in nano-electronics.

Appropriate and accurate simulations of the current flowing in the channel of GFETs are crucial to help the designers in improving and optimizing such devices. The drift-diffusion models represent the simplest way of modeling and are employed in all the standard simulation tools. In the case of GFETs several applications are already present in the literature [4-7] but only compact models are employed for the electrostatic potential. Instead, in [8] a full 2D Poisson equation is included and coupled with the drift-diffusion equations. A new GFET geometry that seems to overcome the zero gap issue and presents a wide off region, robust enough for engineering purposes, has been introduced in [1]. An impor-

(c) The Author(s) 2022. This article is licensed under a Creative Commons Attribution 4.0 International License, which permits use sharing, adaptation, distribution and reproduction in any medium or format, as long as you give appropriate credit to the original author(s) and the source, provide a link to the Creative Commons licence, and indicate if changes were made. The images or other third party material in this article are included in the article's Creative Commons licence, unless indicated otherwise in a credit line to the material. If material is not included in the article's Creative Commons licence and your intended use is not permitted by statutory regulation or exceeds the permitted use, you will need to obtain permission directly from the copyright holder. To view a copy of this licence, visit http://creativecommons.org/licenses/by/4.0/. 
tant issue is to address the importance of the degenerate statistics since often the standard drift-diffusion model based on the Maxwell-Boltzman approximation of the Fermi distribution is used. For such a reason, in this paper we compare the results obtained with the drift-diffusion model in the degenerate case and in the non-degenerate case for the GFET proposed in [1].

The plan of the paper is as follows. In Sect. 2 the mathematical model is described; in Sect. 3 the numerical method is explained; in Sect. 4 numerical results and comparisons are shown.

\section{Mathematical model}

The physical setting is represented by the device proposed in [1], here depicted in Fig. 1. It is made of a sheet of graphene sandwiched between two layers of oxide. Source and drain metallic contacts are placed along all the device thickness while gate contacts are located in the central area of top and bottom oxide.

In order to simulate the current flowing in the channel of the device, we adopt the 1D bipolar stationary drift-diffusion model

$$
\frac{\partial J_{n}}{\partial x}=e R, \quad \frac{\partial J_{p}}{\partial x}=-e R,
$$

$e$ being the (positive) elementary charge. $J_{n}(x)$ and $J_{p}(x)$ are the electron and hole current respectively. $R$ denotes the generation-recombination term. The previous equations are coupled with the 2D Poisson equation for the electrostatic potential in the whole section. The system (1) is set in the interval $\left[x_{1}, x_{4}\right]$ and is augmented with Dirichlet boundary conditions for electron and hole densities as will be explained below.

In this paper, we adopt two different models for the expression of currents, one in the degenerate case and another one for the non-degenerate case.

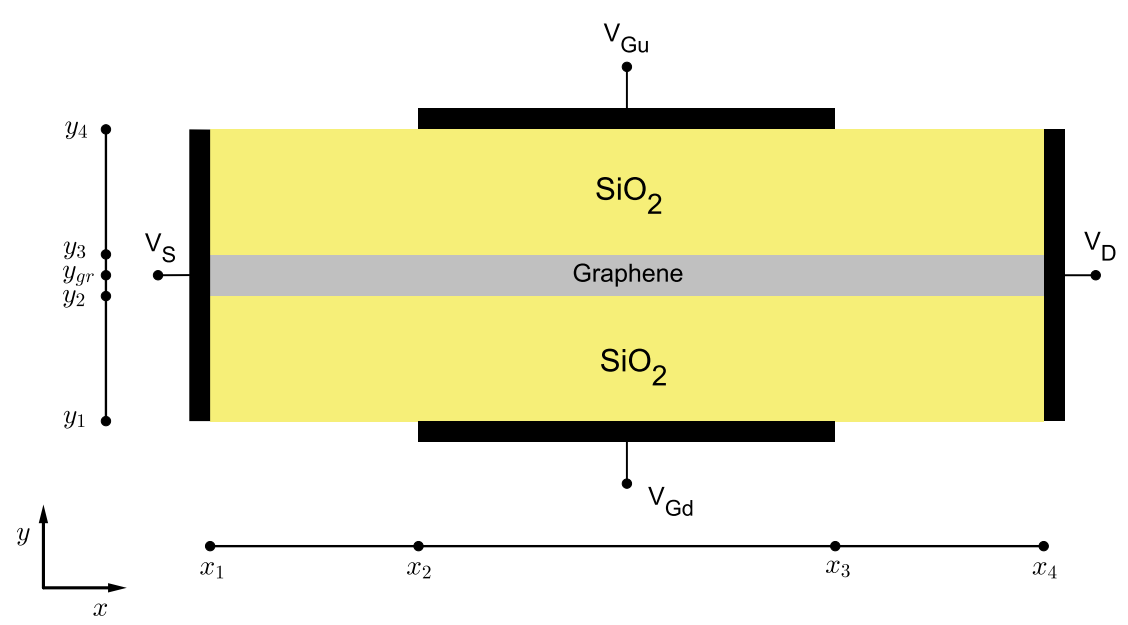

Figure 1 Schematic representation of the simulated GFET. In the direction orthogonal to the $x y$-plane the device is assumed infinitely long 


\subsection{Degenerate case}

The electron and hole current densities are expressed in terms of the quasi-Fermi energies (see for example [7])

$$
J_{n}=\mu_{n} n \frac{\partial \varepsilon_{F}^{(n)}}{\partial x}, \quad J_{p}=\mu_{p} p \frac{\partial \varepsilon_{F}^{(p)}}{\partial x},
$$

where $n$ and $p, \varepsilon_{F}^{(n)}$ and $\varepsilon_{F}^{(p)}, \mu_{n}$ and $\mu_{p}$ are the densities, quasi-Fermi energies and mobilities of electrons and holes, respectively.

The electron and hole densities, $n(x)$ and $p(x)$ respectively, are evaluated as

$$
\begin{aligned}
& n(x)=\frac{g_{s} g_{v}}{(2 \pi)^{2}} \int_{\mathbb{R}^{2}} f_{\mathrm{FD}}\left(\mathbf{k}, \varepsilon_{F}^{(n)}\right) d \mathbf{k}, \quad x \in\left[x_{1}, x_{4}\right], \\
& p(x)=\frac{g_{s} g_{v}}{(2 \pi)^{2}} \int_{\mathbb{R}^{2}} f_{\mathrm{FD}}\left(\mathbf{k},-\varepsilon_{F}^{(p)}\right) d \mathbf{k}, \quad x \in\left[x_{1}, x_{4}\right],
\end{aligned}
$$

with $g_{s}=2$ and $g_{v}=2$ the spin and valley degeneracy. $f_{\mathrm{FD}}$ indicates the Fermi-Dirac distribution

$$
f_{\mathrm{FD}}\left(\mathbf{k}, \varepsilon_{F}\right)=\left[1+\exp \left(\frac{\left(\varepsilon(\mathbf{k})-\varepsilon_{D}\right)-\left(\varepsilon_{F}-\varepsilon_{D}\right)}{k_{B} T}\right)\right]^{-1}
$$

where $\varepsilon_{D}=-e \phi\left(x, y_{\mathrm{gr}}\right)$ is the Dirac energy and $\phi(x, y)$ is the electrical potential, here evaluated on $y=y_{\mathrm{gr}}, y_{\mathrm{gr}}$ being the average $y$-coordinate of the graphene sheet (see Fig. 1). $\varepsilon_{F}$ denotes the Fermi level (in pristine graphene $\varepsilon_{F}=\varepsilon_{D}$ ), $\varepsilon(\mathbf{k})-\varepsilon_{D}=\hbar v_{F}|\mathbf{k}|$ represents the graphene dispersion relation (strictly valid around the Dirac points), which is the same for electrons and holes (see $[2,9,10]), \hbar$ is the reduced Planck constant, $v_{F}$ the Fermi velocity, $k_{B}$ the Boltzmann constant, $T$ the lattice temperature, kept at $300 \mathrm{~K}$ (room temperature). The crystal momentum of electrons and holes is assumed to vary over $\mathbb{R}^{2}$.

We impose Dirichlet boundary conditions on the system (1), (2)

$$
\begin{gathered}
\varepsilon_{F}^{(n)}\left(x_{1}\right)-\varepsilon_{D}\left(x_{1}\right)=\varepsilon_{F}^{(p)}\left(x_{1}\right)-\varepsilon_{D}\left(x_{1}\right)=\alpha \Delta \varepsilon_{F}, \\
\varepsilon_{F}^{(n)}\left(x_{4}\right)-\varepsilon_{D}\left(x_{4}\right)=\varepsilon_{F}^{(p)}\left(x_{4}\right)-\varepsilon_{D}\left(x_{4}\right)=\alpha \Delta \varepsilon_{F} .
\end{gathered}
$$

The quantity $\Delta \varepsilon_{F}$ is the difference of the work functions between metal and graphene. Its value depends on the material the contact is made of. However, only part of the electrons, which the metal can furnish, enters graphene because of quantum reasons (for further explanations see [1]). This gives rise to a kind of contact resistance which is modeled by multiplying $\Delta \varepsilon_{F}$ by the parameter $0<\alpha \leq 1$. In this paper, we use the value $\Delta \varepsilon_{F}=0.25 \mathrm{eV}$ and $\alpha=1$. Simulations with different values of $\alpha$ can be found in [1].

\subsection{Non-degenerate case}

In the presence of low density the Fermi-Dirac distribution can be approximated by the Maxwell-Boltzmann one and the current relations can be written as (see for example [11])

$$
J_{n}=D_{n} \frac{\partial n}{\partial x}-e n \mu_{n} \frac{\partial \phi}{\partial x}, \quad J_{p}=-D_{p} \frac{\partial p}{\partial x}-e p \mu_{p} \frac{\partial \phi}{\partial x}
$$


where $D_{n}=\mu_{n} k_{B} T$ and $D_{p}=\mu_{p} k_{B} T$ are the classical Einstein relations (strictly valid only at equilibrium) for the electron and hole diffusion coefficients, and $\phi$ represents the electrostatic potential along the graphene sheet.

In this case, we impose the following Dirichlet boundary conditions on the system (1), (4)

$$
\begin{aligned}
& n\left(x_{1}\right)=n\left(x_{4}\right)=\frac{g_{s} g_{v}}{(2 \pi)^{2}} \int_{\mathbb{R}^{2}} f_{\mathrm{FD}}\left(\mathbf{k}, \alpha \Delta \varepsilon_{F}\right) d \mathbf{k}, \\
& p\left(x_{1}\right)=p\left(x_{4}\right)=\frac{g_{s} g_{v}}{(2 \pi)^{2}} \int_{\mathbb{R}^{2}} f_{\mathrm{FD}}\left(\mathbf{k},-\alpha \Delta \varepsilon_{F}\right) d \mathbf{k} .
\end{aligned}
$$

\subsection{Generation-recombination and mobilities}

In both cases, for the generation-recombination term $R$ we adopt the Shockley-Read-Hall model, as suggested in [4] by analogy with standard semiconductors

$$
R=\frac{n p-n_{e} p_{e}}{\tau\left(n+p+2 \sqrt{n_{e} p_{e}}\right)}
$$

where $n_{e}$ and $p_{e}$ are the equilibrium electron and hole densities, that is obtained with zero source-drain voltage, and $\tau$ is the generation-recombination relaxation time. For the simulations, in the present paper we have set $\tau=10 \mathrm{ps}$. The qualitative behavior is similar for other values of $\tau$ [1]. Indeed, in [4] an additional term has been included for describing tunnelling effects. It depends on several experimental parameters and its effect should be not very relevant [1]. Here, it is neglected.

The mobility models $\mu_{n}=\mu_{n}(n, E)$ and $\mu_{p}=\mu_{n}(p, E)$ depend in general on the carrier densities $n$ and $p$ and on the electric field $E$, defined along the graphene sheet as

$$
E=-\frac{\partial \phi}{\partial x}
$$

They represent a crucial point for an accurate determination of currents. A mobility model obtained from experimental results has been introduced in [12]. In this paper, we adopt a model deduced by a fitting procedure on extensive simulation of the homogeneous Boltzmann equation solved by a deterministic numerical method [13, 14]. It has been presented in $[15,16]$ and in the general form, including also the presence of a substrate as in the situation adopted here, in [8].

\subsection{Poisson's equation}

The system (1) is coupled with the 2D Poisson equation for the electrostatic potential, solved in the section of the device

$$
\nabla \cdot(\epsilon \nabla \phi)=h(x, y),
$$

where

$$
h(x, y)= \begin{cases}e\left(n(x)-p(x)-n_{\mathrm{imp}}\right) / t_{\mathrm{gr}}, & \text { if }(x, y) \in\left[x_{1}, x_{4}\right] \times\left[y_{2}, y_{3}\right] \\ 0, & \text { otherwise }\end{cases}
$$


The dielectric function $\epsilon$ is given by

$$
\epsilon= \begin{cases}\epsilon_{\mathrm{gr}}, & \text { if } y \in\left[y_{2}, y_{3}\right] \\ \epsilon_{\mathrm{ox}}, & \text { otherwise }\end{cases}
$$

with $\epsilon_{\mathrm{gr}}$ and $\epsilon_{\mathrm{ox}}$ dielectric constants in graphene and oxide respectively. $n_{\mathrm{imp}}$ is the areal density of the impurity charges at the graphene/oxide interface. Since $n$ and $p$ are areal densities, the charge in the graphene layer is considered as distributed in the volume enclosed by the parallelepiped of base the area of the graphene sheet and height $t_{\mathrm{gr}}$.

Equation (6) is augmented with the following boundary conditions

$$
\begin{array}{ll}
\phi=0 & \text { at } y \in\left[y_{1}, y_{4}\right], x=x_{1}, \\
\phi=V_{b} & \text { at } y \in\left[y_{1}, y_{4}\right], x=x_{4}, \\
\phi=V_{G_{d}} & \text { at } y=y_{1}, x \in\left[x_{2}, x_{3}\right], \\
\phi=V_{G_{u}} & \text { at } y=y_{4}, x \in\left[x_{2}, x_{3}\right], \\
\nabla_{\nu} \phi=0 & \text { at the remaining part of } \\
& \text { the boundary. }
\end{array}
$$

Here $V_{b}$ is the bias voltage, $V_{G_{u}}$ is the upper gate-source potential, $V_{G_{d}}$ is the down gatesource potential. $V_{G_{u}}$ and $V_{G_{d}}$ include the (subtracted) flat-band voltages. We have denoted by $\nabla_{v}$ the external normal derivative.

\section{Numerical method}

In order to solve the system (1)-(2) we adopt a finite difference scheme. A uniform mesh of $N_{x}$ grid points and size $\Delta x$ is introduced in $\left[x_{1}, x_{3}\right]$ and for $y=y_{\mathrm{gr}}$. We denote by $u^{i}$ the numerical approximation of a generic function $u(x)$ at the node $i$. First we discretize the equations (1) at the midpoint $x_{i+1 / 2}$ of each interval $\left[x_{i}, x_{i+1}\right]$, that is

$$
\frac{J_{n}^{i+\frac{1}{2}}-J_{n}^{i-\frac{1}{2}}}{\Delta x}=e R^{i}, \quad \frac{J_{p}^{i+\frac{1}{2}}-J_{p}^{i-\frac{1}{2}}}{\Delta x}=-e R^{i} .
$$

Then we discretize the equations for currents (2)

$$
\begin{aligned}
& J_{n}^{i+\frac{1}{2}} \approx \mu_{n}^{i+\frac{1}{2}} n^{i+\frac{1}{2}} \frac{\left(\varepsilon_{F}^{(n)}\right)^{j+1}-\left(\varepsilon_{F}^{(n)}\right)^{j}}{\Delta x} \\
& J_{p}^{i+\frac{1}{2}} \approx \mu_{p}^{i+\frac{1}{2}} p^{i+\frac{1}{2}} \frac{\left(\varepsilon_{F}^{(p)}\right)^{j+1}-\left(\varepsilon_{F}^{(p)}\right)^{j}}{\Delta x}
\end{aligned}
$$

where $\mu_{n}^{i+\frac{1}{2}}, \mu_{p}^{i+\frac{1}{2}}, n^{i+\frac{1}{2}}$ and $p^{i+\frac{1}{2}}$ are obtained by interpolating the same quantities at $i$ and $i+1$ nodes in the midpoint. In this way, an implicit scheme is devised.

We remark that the quantities $\mu_{n}^{i+\frac{1}{2}}, \mu_{p}^{i+\frac{1}{2}}$ depend on $n^{i+\frac{1}{2}}$ and $p^{i+\frac{1}{2}}$ and the latters depend also on $\varepsilon_{F}^{(n)}$ and $\varepsilon_{F}^{(p)}$ through (3). For such a reason, the numerical solution is obtained by the following iterative procedure.

We denote by $u^{(r)}$ the value of the function $u$ at the step $r$. 
1. $n^{(1)}=p^{(1)}=n_{i}$ for each $x \in\left[x_{1}, x_{4}\right]$, where $n_{i}$ is the intrinsic graphene concentration, and get $\varphi^{(1)}$ solving the Poisson equation (6)-(7).

2. For each $r>1$ :

(a) find $\left(\varepsilon_{F}^{(n)}\right)^{(r)}$ and $\left(\varepsilon_{F}^{(p)}\right)^{(r)}$ solving the drift-diffusion equations (1)-(2) with the electrostatic potential $\varphi^{(r-1)}$;

(b) calculate $n^{(r)}$ and $p^{(r)}$ through (3);

(c) solve the Poisson equation (6)-(7) with densities $n^{(r)}$ and $p^{(r)}$ getting $\varphi^{(r)}$.

3. Iterate step 2 until convergence.

In order to improve the convergence, Pulay's method has been employed as in [6]. The difference between two iterations is measured as the relative variation of the current

$$
e_{n}=\frac{\left\|J_{n}^{(r+1)}-J_{n}^{(r)}\right\|}{\left\|J_{n}^{(r+1)}\right\|}, \quad e_{p}=\frac{\left\|J_{p}^{(r+1)}-J_{p}^{(r)}\right\|}{\left\|J_{p}^{(r+1)}\right\|},
$$

where $J_{n}^{(r)}$ and $J_{p}^{(r)}$ are the electron and hole currents at the $r$ th iteration. As stopping criterion $e_{n}$ and $e_{p}$ less than $10^{-4}$ has been required. Here $\|\cdot\|$ denotes the supremum norm.

The system (1), (4) is solved by the Scharfetter-Gummel scheme in the stationary case with the aid of Pulay's method, even in this case. The discretization is similar to the time dependent one and the interested reader is referred to [8].

To solve the Poisson equation numerically a uniform two-dimensional mesh is adopted. It is discretized by finite differences at the internal points with the standard five points stencil [8].

\section{Simulation results}

In this section, we show the results of numerical simulations of the device of Fig. 1. We have set the length $100 \mathrm{~nm}$, the width of the lower and upper oxide $\left(\mathrm{SiO}_{2}\right) 10 \mathrm{~nm}$. The graphene thickness $t_{\mathrm{gr}}$, including also the double graphene/oxide interface, is set to $1 \mathrm{~nm}$. The gate contacts are long $50 \mathrm{~nm}$. The lateral (source and drain) contacts are long $21 \mathrm{~nm}$. The two gate potentials are both set equal to the same value $V_{G}$ in the simulated cases.

In Eqs. (6)-(7) we adopt the values $\epsilon_{\mathrm{gr}}=3.3 \epsilon_{0}$ and $\epsilon_{\mathrm{ox}}=3.6 \epsilon_{0}$, where $\epsilon_{0}$ is the dielectric constant in the vacuum, and $n_{\mathrm{imp}}=2.5 \cdot 10^{3} \mu \mathrm{m}^{-2}$.

We considered a mesh of 41 grid points along the $x$-direction and 23 grid points along the $y$-direction. In the graphene layer a single row of 39 nodes has been employed.

In Fig. 2 we show, for several drain-source voltages $V_{b}$ and gate voltages $V_{G}$, the characteristic curves of the simulated GFET obtained with the model (1), (2). In Fig. 3 the same curves are reported adopting the model (1), (4). There are evident qualitative and quantitative differences, in particular at the lower bias voltages considered in the simulations. For example in the case $V_{b}=0.1 \mathrm{~V}$, when $V_{G}=1.5$ we have a difference of about $74 \%$. The discrepancy reduces at higher voltage but it is clear that the model (1), (4) overestimates the current anyway.

A comparison of electron densities with both models is shown in Fig. 4. The simulations are obtained with $V_{b}=0.2 \mathrm{~V}$ and $V_{G}=-1.5$ and $1.5 \mathrm{~V}$. In Fig. 5 a similar plot is presented for hole densities. The differences are less evident with respect to the currents. This seems to indicate that the crucial point is a reduction of the mean velocity when the degeneracy effects are taken into account.

For the sake of completeness also the electrostatic potential is depicted (Fig. 6) in the same cases. For the electrostatic potential no significant differences are obtained. 

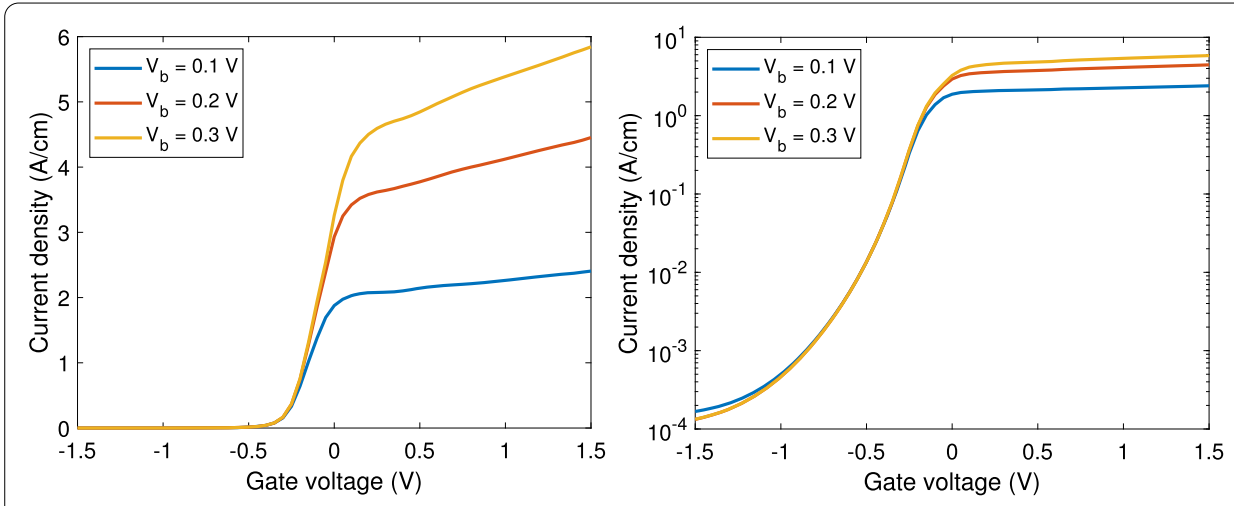

Figure 2 Characteristic curves of the simulated GFET in linear scale (left) and logarithmic one (right). The adopted model is (1), (2)
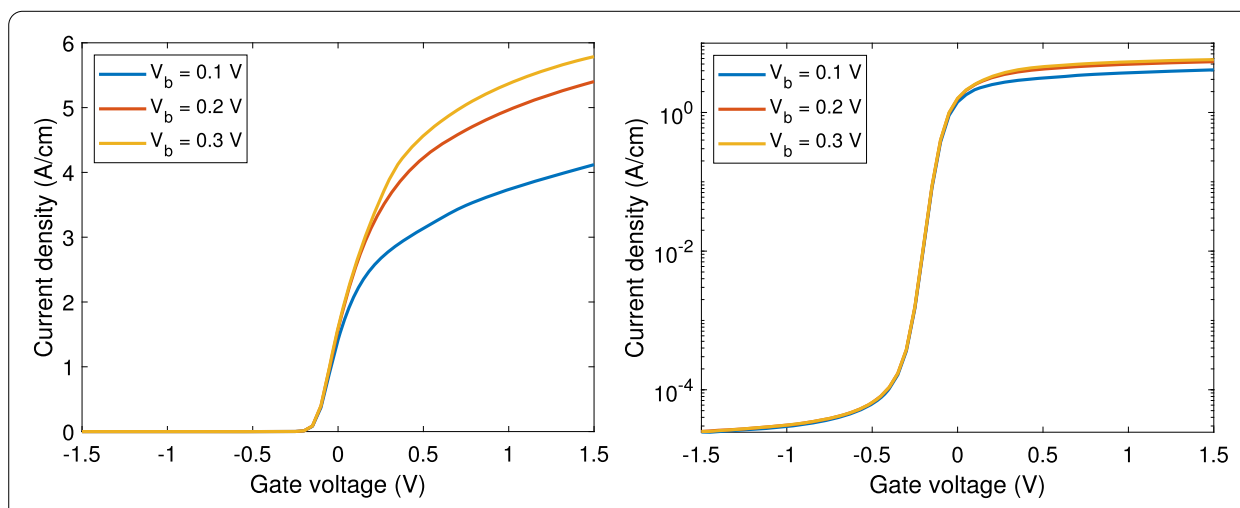

Figure 3 Characteristic curves of the simulated GFET in linear scale (left) and logarithmic one (right). The adopted model is (1), (4)
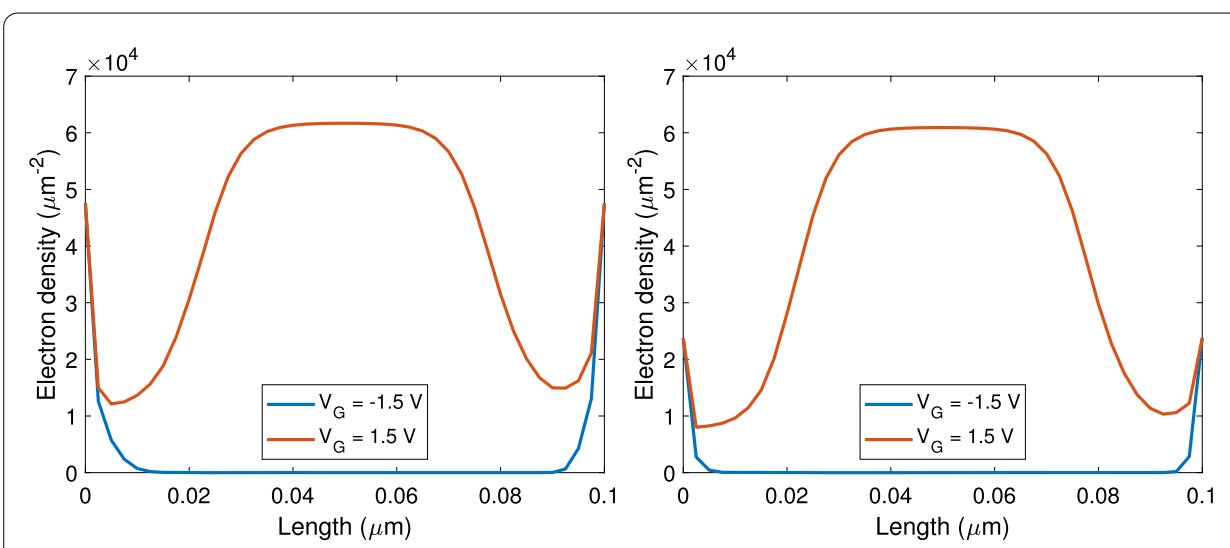

Figure 4 Electron density of the simulated GFET in the case $V_{b}=0.2 \mathrm{~V}$ and $V_{G}=-1.5$ and $1.5 \mathrm{~V}$. On the left the adopted model is (1), (2); on the right it is (1), (4)

In order to better understand the origin of the discrepancy between the degenerate and non-degenerate cases, we look closer to the models. By observing that $d \mathbf{k}=\frac{\left(\varepsilon-\varepsilon_{D}\right) d\left(\varepsilon-\varepsilon_{D}\right) d \varphi}{\left(\hbar \nu_{F}\right)^{2}}$, with $\varphi$ azimuthal angle of $\mathbf{k}$, the electron and hole densities can be written in terms of the 

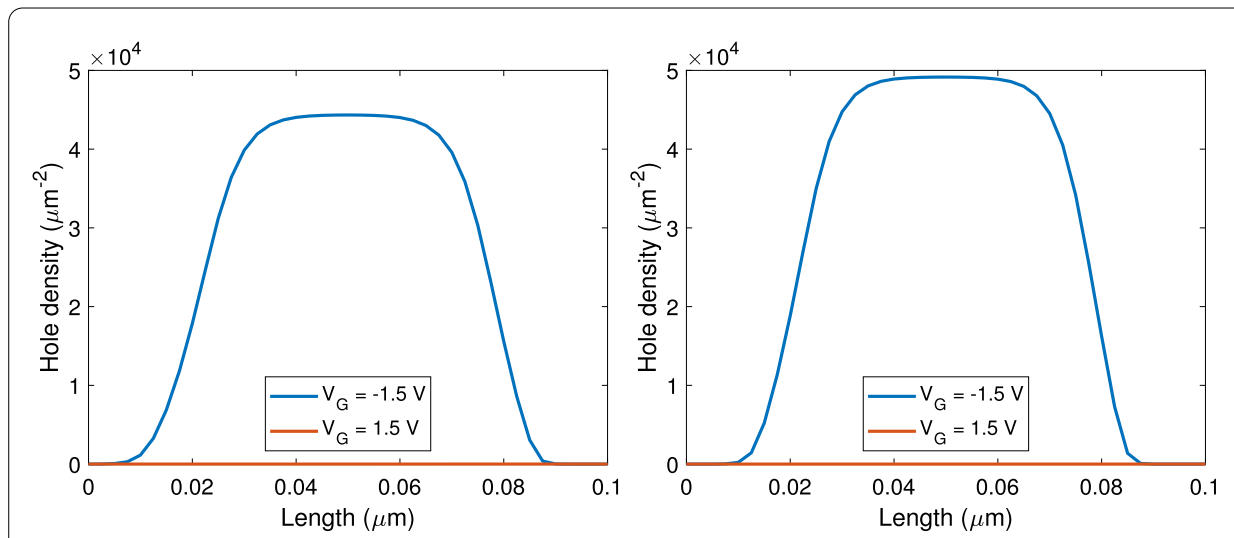

Figure 5 Hole density of the simulated GFET in the case $V_{b}=0.2 \mathrm{~V}$ and $V_{G}=-1.5$ and $1.5 \mathrm{~V}$. On the left the adopted model is (1), (2); on the right it is (1), (4)

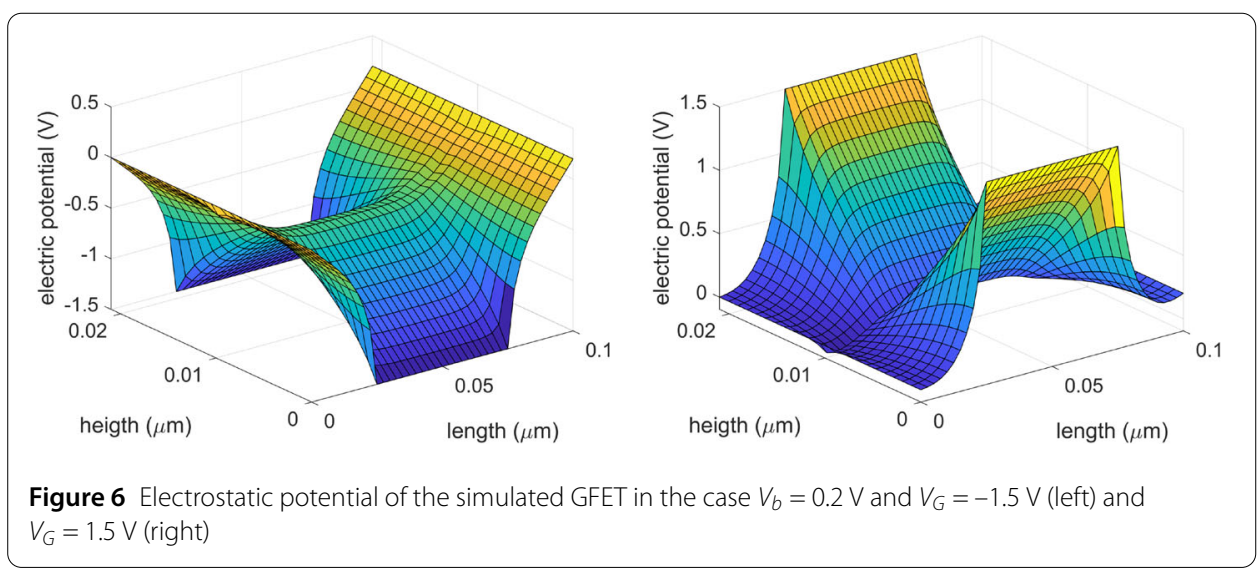

Fermi integrals of order $k$

$$
\mathcal{F}_{k}(z)=\frac{1}{\Gamma(k+1)} \int_{0}^{+\infty} \frac{\chi^{k}}{1+e^{\chi-z}} d \chi
$$

with $\Gamma(x)$ Euler gamma function, as follows

$$
\begin{aligned}
& n(x)=\frac{2}{\pi}\left(\frac{k_{B} T}{\hbar v_{F}}\right)^{2} \mathcal{F}_{1}\left(\frac{\varepsilon_{F}^{(n)}(x)-\varepsilon_{D}(x)}{k_{B} T}\right), \\
& p(x)=\frac{2}{\pi}\left(\frac{k_{B} T}{\hbar v_{F}}\right)^{2} \mathcal{F}_{1}\left(-\frac{\varepsilon_{F}^{(p)}(x)-\varepsilon_{D}(x)}{k_{B} T}\right) .
\end{aligned}
$$

Here $\eta^{(n)}(x)=\varepsilon_{F}^{(n)}(x)-\varepsilon_{D}(x)$ and $\eta^{(p)}(x)=\varepsilon_{F}^{(p)}(x)-\varepsilon_{D}(x)$ are the chemical energy for electrons and holes respectively. Therefore

$$
J_{n}=\mu_{n} n \nabla \varepsilon_{F}^{(n)}=\mu_{n} n \nabla\left(\eta^{(n)}+\varepsilon_{D}\right)=\mu_{n} n \nabla \eta^{(n)}-e \mu_{n} n \nabla \phi .
$$


The second term is the standard drift one. By observing that $\mathcal{F}_{1}(z)$ is a strict monotone function and recalling the well known properties of the Fermi integral

$$
\frac{d \mathcal{F}_{k}(z)}{d z}=\mathcal{F}_{k-1}(z), \quad k=1,2, \ldots \quad \text { and } \quad \mathcal{F}_{0}(z)=\ln (1+\exp z)
$$

the first term is given by

$$
\mu_{n} n \nabla \eta^{(n)}=\mu_{n} n \frac{d \eta^{(n)}}{d n} \nabla n=\frac{\mu_{n} n}{\frac{d n}{d \eta^{(n)}}} \nabla n
$$

from which the electron diffusion coefficient in the degenerate case

$$
D_{n}^{d}=\frac{\mu_{n} n}{\frac{d n}{d \eta^{(n)}}}=\mu_{n} k_{B} T \frac{\mathcal{F}_{1}\left(\frac{\eta^{(n)}}{k_{B} T}\right)}{\ln \left[1+\exp \left(\frac{\eta^{(n)}}{k_{B} T}\right)\right]}=D_{n} \frac{\mathcal{F}_{1}\left(\frac{\eta^{(n)}}{k_{B} T}\right)}{\ln \left[1+\exp \left(\frac{\eta^{(n)}}{k_{B} T}\right)\right]} .
$$

Similarly the diffusion coefficient of holes in the degenerate case is related to that in the non-degenerate case by

$$
D_{p}^{d}=D_{p} \frac{\mathcal{F}_{1}\left(\frac{-\eta^{(p)}}{k_{B} T}\right)}{\ln \left[1+\exp \left(\frac{-\eta(p)}{k_{B} T}\right)\right]}
$$

In Fig. 7 the ratios $r_{n}=\frac{D_{n}^{d}}{D_{n}}$ and $r_{p}=\frac{D_{p}^{d}}{D_{p}}$ are plotted along the graphene in the simulated cased considered above. For electrons $r_{n}$ is considerably different from one near the contacts while $r_{p}$ is in a significant way greater than one in the channel. Anyway there is a clear evidence that the classical Einstein relations for the diffusion coefficients are not adequate in the simulation of the considered device. This casts serious doubts on the use of the non-degenerate drift-diffusion models in GFET.
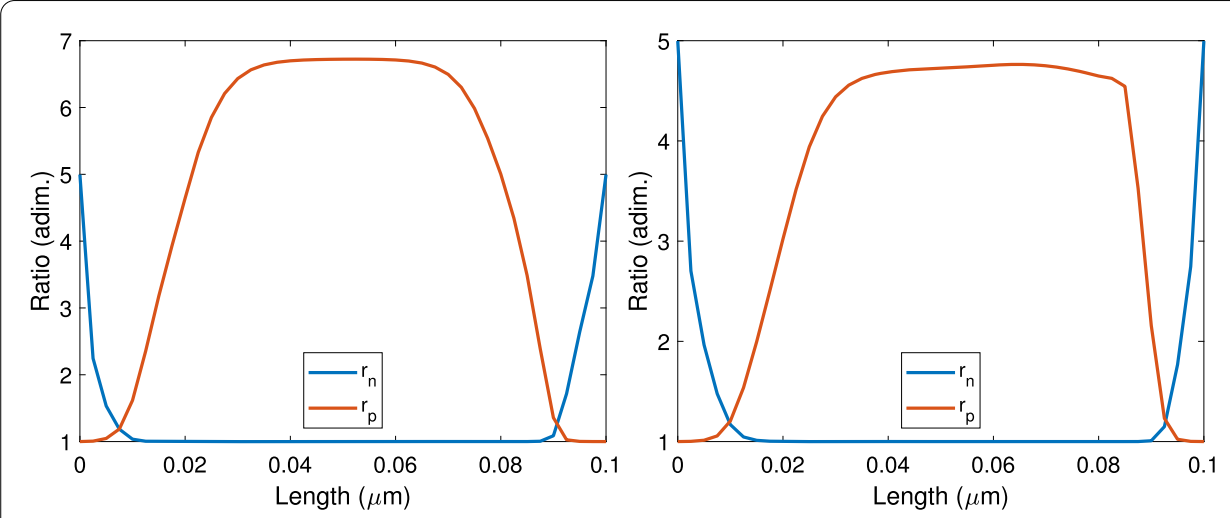

Figure 7 Ratios of the degenerate and non-degenerate diffusion coefficients in the case $V_{b}=0.2 \mathrm{~V}$ and $V_{G}=-1.5 \mathrm{~V}$ (left) and $V_{G}=1.5 \mathrm{~V}$ (right) 


\section{Conclusions}

The degenerate and non-degenerate drift-diffusion models have been compared in the case of charge transport in graphene by simulating the GFET proposed in [1]. Major differences are observed regarding the characteristic curves making the drift-diffusion models based on the Maxwell-Boltzmann statistics not adequate in the considered device. A more in-depth analysis shows that the root of the failure is in the diffusion coefficient which is very different in the degenerate and non-degenerate case. The Einstein relation is no longer valid in GFET.

A further step forward could be the inclusion of the thermal effects (see for example [17-20]) or quantum correction [21-23].

\section{Acknowledgements}

Not applicable.

\section{Funding}

The authors acknowledge the support from INdAM (GNFM) and from Università degli Studi di Catania, Piano della Ricerca 2020/2022 Linea di intervento 2 "QICT". GN acknowledges the financial support from Progetto Giovani GNFM 2020

"Trasporto di cariche e fononi in strutture a bassa dimensione".

\section{Abbreviations}

FET, field effect transistor; GFET, graphene field effect transistor; MOSFET, metal oxide semiconductor field effect transistor.

Availability of data and materials

Not applicable.

\section{Declarations}

\section{Competing interests}

The authors declare that they have no competing interests.

\section{Authors' contributions}

Both authors contributed equally to the writing of the manuscript. All authors read and approved the final manuscript.

\section{Publisher's Note}

Springer Nature remains neutral with regard to jurisdictional claims in published maps and institutional affiliations.

Received: 9 November 2021 Accepted: 13 January 2022 Published online: 24 January 2022

\section{References}

1. Nastasi G, Romano V. An efficient GFET structure. IEEE Trans Electron Devices. 2021;68:4729-34. https://doi.org/10.1109/TED.2021.3096492.

2. Castro Neto AH, Guinea F, Peres NMR, Novoselov KS, Geim AK. The electronic properties of graphene. Rev Mod Phys. 2009;81:109-62. https://doi.org/10.1103/RevModPhys.81.109.

3. Schwierz F. Graphene transistors. Nat Nanotechnol. 2010:5:487-96. https://doi.org/10.1038/nnano.2010.89.

4. Ancona MG. Electron transport in graphene from a diffusion-drift perspective. IEEE Trans Electron Devices. 2010;57:681-9. https://doi.org/10.1109/TED.2009.2038644.

5. Jiménez D, Moldovan O. Explicit drain-current model of graphene field-effect transistors targeting analog and radio-frequency applications. IEEE Trans Electron Devices. 2011;58:4049-52. https://doi.org/10.1109/TED.2011.2163517.

6. Feijoo PC, Jimńez D, Cartoixà X. Short channel effects in graphene-based field effect transistors targeting radio-frequency applications. 2D Mater. 2016;3:025036. https://doi.org/10.1088/2053-1583/3/2/025036.

7. Champlain JG. A first principles theoretical examination of graphene-based field effect transistors. J Appl Phys. 2011;109:084515. https://doi.org/10.1063/1.3573517.

8. Nastasi G, Romano V. A full coupled drift-diffusion-Poisson simulation of a GFET. Commun Nonlinear Sci Numer Simul. 2020;87:105300. https://doi.org/10.1016/j.cnsns.2020.105300.

9. Jacoboni C. Theory of electron transport in semiconductors. 1st ed. Berlin: Springer; 2010.

10. Kittel C. Introduction to solid state physics. 7th ed. Hoboken: Wiley; 2005.

11. Selberherr G. Analysis and simulation of semiconductor devices. Vienna: Springer; 1984

12. Dorgan VE, Bae M-H, Pop E. Mobility and saturation velocity in graphene on $\mathrm{SiO}_{2}$. Appl Phys Lett. 2010;97:082112. https://doi.org/10.1063/1.3483130.

13. Coco M, Majorana A, Romano V. Cross validation of discontinuous Galerkin method and Monte Carlo simulations of charge transport in graphene on substrate. Ric Mat. 2017;66:201-20. https://doi.org/10.1007/s11587-016-0298-4. 
14. Majorana A, Nastasi G, Romano V. Simulation of bipolar charge transport in graphene by using a discontinuous Galerkin method. Commun Comput Phys. 2019;26:114-34. https://doi.org/10.4208/cicp.OA-2018-0052.

15. Majorana A, Mascali G, Romano V. Charge transport and mobility in monolayer graphene. J Math Ind. $2016 ; 7: 4$. https://doi.org/10.1186/s13362-016-0027-3.

16. Nastasi G, Romano V. Improved mobility models for charge transport in graphene. Commun Appl Ind Math. 2019:10:41-52. https://doi.org/10.1515/caim-2019-0011.

17. Coco M, Romano V. Simulation of electron-phonon coupling and heating dynamics in suspended monolayer graphene including all the phonon branches. J Heat Transf. 2018;140:092404. https://doi.org/10.1115/1.4040082.

18. Mascali G. A hydrodynamic model for silicon semiconductors including crystal heating. Eur J Appl Math. 2015;26:447-96. https://doi.org/10.1017/S0956792515000157.

19. Mascali G, Romano V. Charge transport in graphene including thermal effects. SIAM J Appl Math. 2017;77:593-613. https://doi.org/10.1137/15M1052573.

20. Mascali G, Romano V. Exploitation of the maximum entropy principle in mathematical modeling of charge transport in semiconductors. Entropy. 2017;19:36. https://doi.org/10.3390/e19010036.

21. Luca L, Romano V. Quantum corrected hydrodynamic models for charge transport in graphene. Ann Phys. 2019;406:30-53. https://doi.org/10.1016/j.aop.2019.03.018.

22. Barletti L, Cintolesi C. Derivation of isothermal quantum fluid equations with Fermi-Dirac and Bose-Einstein statistics. J Stat Phys. 2012;148:353-86. https://doi.org/10.1007/s10955-012-0535-5.

23. Camiola VD, Luca L, Equilibrium RV. Wigner function for fermions and bosons in the case of a general energy dispersion relation. Entropy. 2020;22:1023. https://doi.org/10.3390/e22091023.

\section{Submit your manuscript to a SpringerOpen ${ }^{\circ}$ journal and benefit from:}

- Convenient online submission

- Rigorous peer review

Open access: articles freely available online

- High visibility within the field

- Retaining the copyright to your article

Submit your next manuscript at $\boldsymbol{\Delta}$ springeropen.com 